life outcomes. The study is enrolling and will have sites in the USA, Europe, Japan, Latin America, Taiwan, Singapore, and South Korea.

Results Not applicable.

Conclusions Not applicable.

\section{EPV256/\#150 PREOPERATIVE FRAILTY ASSESSMENT IN PATIENTS UNDERGOING GYNECOLOGIC ONCOLOGY SURGERY: A SYSTEMATIC REVIEW}

${ }^{1} \mathrm{~V}$ Di Donato, ${ }^{2} \mathrm{G}$ Caruso, ${ }^{3} \mathrm{G}$ Bogani, ${ }^{2} \mathrm{G}$ Perniola, ${ }^{2}$ Palaia, ${ }^{4} \mathrm{~F}$ Plotti, ${ }^{4} \mathrm{R}$ Angioli, ${ }^{5} \mathrm{~L}$ Muzii, ${ }^{4} \mathrm{P}$ Benedetti Panici. 'Umberto I, 'Sapienza' University of Rome, Department of Maternal and Child Health and Urological Sciences, Rome, Italy; ${ }^{2}$ Sapienza University of Rome, Department of Maternal and Child Health and Urological Sciences, Rome, Italy; ${ }^{3}$ IRCCS National Cancer Institute, Department of Gynecologic Oncology, Milano, Italy; ${ }^{4}$ Campus Biomedico, Gynecologic Oncology, Rome, Italy; ${ }^{5}$ Sapienza University, Gynecologic Oncology, Rome, Italy

\subsection{6/ijgc-2021-IGCS.327}

Objectives The aim of the present article was to discuss currently available evidence on the impact of frailty assessment on adverse postoperative outcomes and survival in patients undergoing surgery for gynecological cancer.

Methods Systematic search of Medline (PubMed) and Embase databases until September 30, 2020. Key inclusion criteria were: (1) randomized or observational studies; (2) patients undergoing non-emergent surgery for gynecological malignancies; (3) preoperative frailty assessment.

Results Through the process of evidence acquisition, twelve studies including 85,672 patients were selected and six tools were evaluable: 30-item frailty index, 40-item frailty index, modified frailty index (mFI), John Hopkins Adjusted Clinical Groups index, Fried frailty criteria, Driver's tool. The prevalence of frailty varied roughly from $6.1 \%$ to $60 \%$ across different series included. The mFI was the most adopted and predictive instrument. Pooled results underlined that frail patients were more likely to develop 30-day postoperative complications (OR, 4.16; 95\% CI, 1.49-11.65; p=0.007), non-home discharge (OR，4.41; 95\% CI, 4.09-4.76; $\mathrm{p}<0.001$ ), ICU admission (OR:3.99; 95\% CI, 3.76-4.24; $\mathrm{p}<0.001$ ) than the non-frail counterpart. Additionally, frail patients experienced worse oncologic outcomes (disease-free and overall survivals) than non-frail patients.

Conclusions The present systematic review demonstrated that preoperative frailty assessment among gynecologic oncology patients is essential to predict adverse outcomes and tailor a personalized treatment. The $\mathrm{mFI}$ appeared as the most used and feasible tool in daily practice, suggesting that tailored therapeutic strategies should be considered for patients with 3 or more frailty-defining items.

\section{EPV257/\#152 SURGICAL SITE INFILTRATION VERSUS TRANSVERSUS ABDOMINIS PLANE BLOCK OF LIPOSOMAL BUPIVACAINE AFTER MIDLINE VERTICAL LAPAROTOMY FOR GYNECOLOGIC MALIGNANCY: A PROSPECTIVE RANDOMIZED CONTROLLED TRIAL}

\footnotetext{
${ }^{1} \mathrm{~A}$ Moon*, ${ }^{1} \mathrm{~V}$ andikyan, ${ }^{2} \mathrm{R}$ Agarwal, ${ }^{2} \mathrm{~S}$ Stroever, ${ }^{3} \mathrm{D}$ Misita, ${ }^{1} \mathrm{~A}$ Laibangyang, ${ }^{1} \mathrm{D}$ Doo, ${ }^{1} \mathrm{~L}$ Chuang. 'Nuvance Health, Obstetrics, Gynecology and Reproductive Biology, Division of Gynecologic Oncology, Danbury, USA; ${ }^{2}$ Nuvance Health, Research and Innovation, Danbury, USA; ${ }^{3}$ Nuvance Health, Anesthesiology, Danbury, USA
}

10.1136/ijgc-2021-IGCS.328
Objectives Surgical site infiltration (SSI) and transversus abdominis plane (TAP) block are postoperative analgesic techniques. Liposomal bupivacaine may prolong analgesic effects. We hypothesize that surgical site infiltration of liposomal bupivacaine will reduce opioid consumption in the 48 -hour postoperative period compared to TAP block.

Methods A single blind randomized controlled trial comparing surgical site infiltration of liposomal bupivacaine versus TAP block with liposomal bupivacaine after midline vertical laparotomy in patients with suspected or known gynecologic malignancy. Negative binomial regression was used to estimate the differences in total morphine milligram equivalent (MME) use between groups. Multivariable linear regression of pain scores on visual analog scale $0-10$ was used at each time interval (2, $6,12,24$, and 48 hours postoperatively) while controlling for medication use and age.

Results Of 43 patients, 22 received SSI and 21 received TAP block. Mean age was $57.8(\mathrm{SD}=11.50)$. There were no significant differences in demographics, incision length, surgery duration or pathology between groups. After controlling for age and BMI, there was not a statistically significant difference in total MME between the treatment groups $(\beta=-0.17,95 \%$ $\mathrm{CI}=-0.77,0.43, \mathrm{p}=0.59)$. There were no statistically significant differences in pain scores (both resting and exertion) at all time points after controlling for age and pain medication utilization.

Conclusions Surgical site infiltration of liposomal bupivacaine did not reduce opioid use and did not decrease pain scores within 48 hours after surgery compared to TAP block after midline vertical laparotomy for gynecologic cancer.

\section{EPV258/\#285 IMPROVING THE RATES OF SAME DAY DISCHARGE IN ROBOTIC SURGERY PATIENTS - A GYNECOLOGIC ONCOLOGY QUALITY IMPROVEMENT PROJECT}

1J Mateshaytis*, ${ }^{2} \mathrm{~S}$ Pin, ${ }^{2} \mathrm{H}$ Steed, ${ }^{2} \mathrm{M}$ Brawner. 'University of Calgary, Gynecologic Oncology, Calgary, Canada; ${ }^{2}$ University of Alberta, Gynecologic Oncology, Edmonton, Canada

\subsection{6/ijgc-2021-IGCS.329}

Objectives In appropriately selected gynecologic oncology (GO) patients, robotic or laparoscopic surgery is a preferred approach (faster recovery, fewer complications, shorter hospital stay). Furthermore, same day discharge (SDD) is safe and effective in these patients. Evidence suggests no increased rates of readmission, or complications for SDD compared with overnight observation. We pursued a Quality Improvement (QI) project aimed at increasing our rate of SDD by $50 \%$ by June 2021 in GO robotic surgery patients.

Methods This QI initiative is based upon the Institute for Healthcare Improvement's Model for Improvement. The study is an interrupted time series study. Baseline data assessment determined the rate of SDD and potential root causes for failed SDD. For each intervention (addressing a root cause), Plan-Do-Study-Act cycles were conducted. Outcome, process, and balancing measures were collected prospectively.

Results Four simple interventions were selected for implementation: 1) setting SDD as the default discharge plan, 2) providing a physician discharge order on the patient chart, 3) removing the foley catheter in the OR, 4) developing comprehensive standardized perioperative patient education materials. The rate of SDD was improved from $28.8 \%$ (baseline) to 
$69.4 \%$ following the changes. There was no increased 30-day rate of readmission ( $0 \%$ SDD vs $1.3 \%$ all robotic cases) or presentation to the emergency department (1.9\% SDD vs $3.8 \%$ all robotic cases) following implementation of the interventions.

Conclusions Local rates of SDD can be improved with simple interventions targeting disposition planning, foley catheter removal and managing patient expectations. These interventions may be easily applicable to other GO programs.

\section{EPV259/\#288 PATIENT OUTCOMES AND ADHERENCE TO AN ENHANCED RECOVERY PATHWAY FOR OPEN GYNECOLOGIC ONCOLOGY SURGERY: A 5-YEAR SINGLE CENTER EXPERIENCE}

1J Hayek, ${ }^{2} \mathrm{~A}$ Zorrilla-Vaca, ${ }^{3} \mathrm{~L}$ Meyer, ${ }^{2} \mathrm{G}$ Mena, ${ }^{2} \mathrm{~L}$ Lasala, ${ }^{3} \mathrm{M}$ Iniesta, ${ }^{3} \mathrm{~T}$ Suki, ${ }^{4} \mathrm{~K}$ Cain, ${ }^{3} \mathrm{~J}$ Garcia-Lopez, ${ }^{3} \mathrm{P}$ Ramirez. 'Hofstra/Northwell at Staten Island University Hospital, Obstetrics and Gynecology, New York, USA; ${ }^{2}$ The University of Texas MD anderson Cancer Center, Department of Anesthesiology, Houston, USA; ${ }^{3}$ University of Texas MD anderson Cancer Center, Gynecologic Oncology and Reproductive Medicine, Houston, USA; ${ }^{4}$ The University of Texas MD anderson Cancer Center, Division of Pharmacy, Houston, USA

\subsection{6/ijgc-2021-IGCS.330}

Objectives This study evaluates compliance with Enhanced Recovery After Surgery (ERAS) protocol for open gynecologic oncologic surgery at a tertiary center and relationship between levels of compliance and perioperative outcomes.

Methods Our retrospective cohort study included 1879 patients between November 2014 and December 2020. Two groups were identified based on compliance level ( $<80 \%$ versus $\geq 80 \%$ ). Our primary outcomes were 30-day readmission, reoperation, length of stay, and postoperative complications. We also assessed compliance with each ERAS item over time (P1: 2014-2016, P2: 2017-2018, P3: 2019-2020) categorizing patients according to date of surgery. Values were compared between P3 and P1. Multivariable logistic regression analyses were performed to evaluate associations between high compliance and perioperative outcomes.

Results Overall compliance was 74\% (95\%, CI 71.9-78.2). Compliance with ERAS $>80 \%$ was associated with lower Clavien-Dindo grades II (OR 0.74, 95\%CI 0.59-0.93), III (OR 0.55, 95\%CI 0.33-0.93), and V (OR 0.08, 95\%CI 0.010.60 ) complication rates, readmission rates (OR $0.61 ; 95 \% \mathrm{CI}$ $0.43-0.88$ ) and shorter length of stay (OR 0.59; 95\%CI $0.47-0.75)$. Preoperatively, there was increased adherence to counseling $(50 \%, \mathrm{p}=0.01)$, optimization $(21 \%, \mathrm{p}=0.02)$, and carbohydrate-loading $(74 \%, \mathrm{p}=0.02)$. Intraoperatively, use of short-acting anesthetics and adherence to avoiding abdominal drainage $(7 \%, \mathrm{p}=0.04)$ increased. Compliance with goal directed fluid therapy $(16 \%, p=0.04)$ and normothermia $(8 \%$, $\mathrm{p}=0.03)$ decreased. Postoperatively, there was increased compliance with avoiding saltwater overload $(8 \%, \mathrm{p}=0.02)$ and multimodal analgesia $(5 \%, \mathrm{p}=0.02)$.

Conclusions Compliance $(>80 \%)$ with ERAS is associated with lower complication rates, 30-day readmissions, and shorter length of stay without impacting reoperation rates and postoperative opioid use.

\section{EPV260/\#451 SCHWANNOMA OF THE PUDENDAL NERVE - ANATOMICAL CONSIDERATIONS IN THE APPROACH TO SURGICAL MANAGEMENT OF THIS RARE PATHOLOGY}

${ }^{1} S$ Smyth*, ${ }^{2} \mathrm{~L}$ Cogswell, ${ }^{3}$ S Dhar, ${ }^{1} \mathrm{H}$ Soleymani Majd. ${ }^{1}$ Oxford University Hospitals NHS Foundation Trust, Gynaecological Oncology, Oxford, UK; ${ }^{2}$ Oxford University Hospitals NHS Foundation Trust, Plastic Surgery, Oxford, UK; ${ }^{3}$ Oxford University Hospitals NHS Foundation Trust, Histopathology, Oxford, UK

\subsection{6/ijgc-2021-IGCS.331}

Objectives Schwannomas are uncommon, benign, indolent, nerve sheath tumours with low malignant potential; commonly affecting the nerves of the head, neck, mediastinum and extremities. Two pudendal cases have been reported. Their deep, complex location challenges resection - via the ischiorectal fossa in our case.

Methods A 24-year-old female reported an enlarging left perineal mass over eight years; associated with numbness and sexual dysfunction. This was $7 \times 5 \mathrm{~cm}$ on examination, distal to Alcock's canal, with no vaginal, rectal or anal sphincter involvement. It was defined and mildly FDG-avid on imaging. Biopsies confirmed schwannoma. She reports no neurological deficit or evidence of recurrence following resection.

Results Alcock's canal is directly accessed via the ischiorectal fossa with minimal pelvic muscle and ligament disruption. The pudendal nerve arises from the S2-4 sacral nerve and travels forward laterally in the pelvis within this obturator internus fascial sheath. It has both motor and sensory functions. The ischiorectal fossa is a pyramidal space lateral to the anal canal and below the pelvic diaphragm. It contains the internal pudendal and inferior rectal vessels and nerves.

Conclusions Pudendal nerve schwannomas are rare, arising from a single non-functioning sensory fascicle. Following

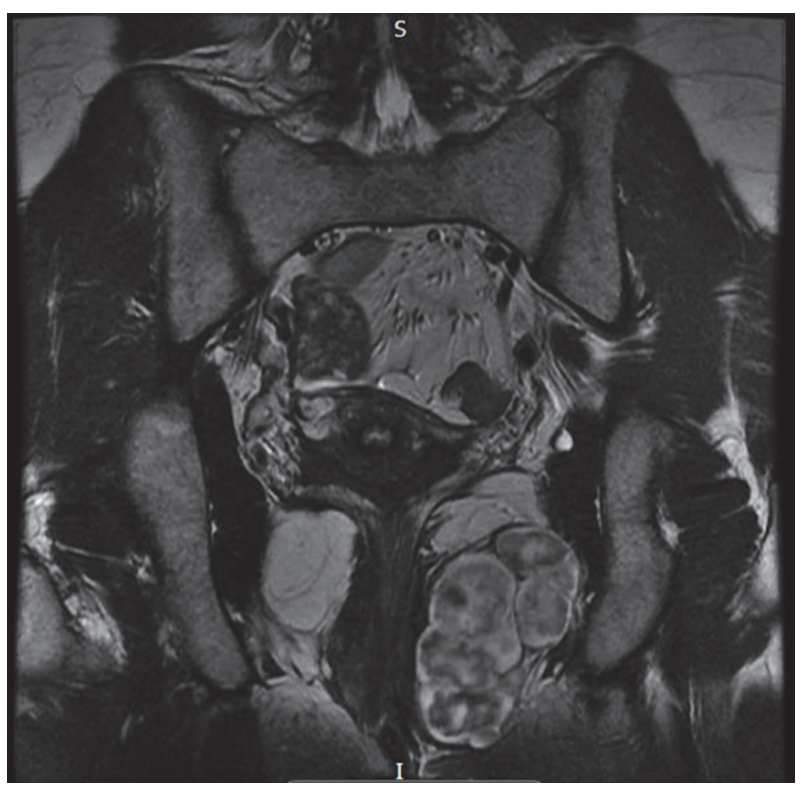

Abstract EPV260/\#451 Figure 1 